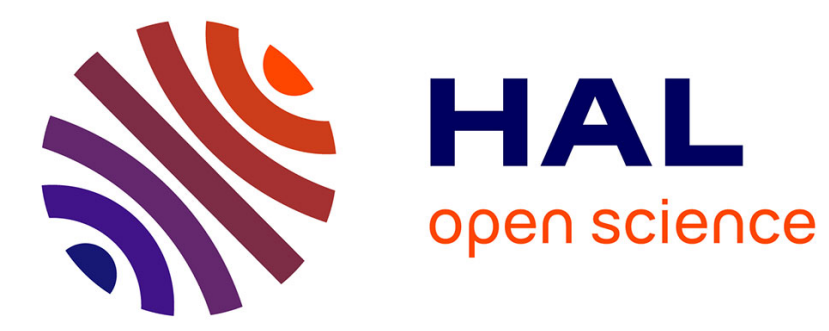

\title{
Unidirectional Direct Load Control through Smart Plugs
}

Giovanni Neglia, Giuseppe Di Bella, Laura Giarrè, Ilenia Tinnirello

\section{To cite this version:}

Giovanni Neglia, Giuseppe Di Bella, Laura Giarrè, Ilenia Tinnirello. Unidirectional Direct Load Control through Smart Plugs. IEEE 53rd Annual Conference on Decision and Control (CDC 2014), Dec 2014, Los Angeles, United States. hal-01094640

\section{HAL Id: hal-01094640 \\ https://hal.inria.fr/hal-01094640}

Submitted on 12 Dec 2014

HAL is a multi-disciplinary open access archive for the deposit and dissemination of scientific research documents, whether they are published or not. The documents may come from teaching and research institutions in France or abroad, or from public or private research centers.
L'archive ouverte pluridisciplinaire HAL, est destinée au dépôt et à la diffusion de documents scientifiques de niveau recherche, publiés ou non, émanant des établissements d'enseignement et de recherche français ou étrangers, des laboratoires publics ou privés. 


\title{
Unidirectional Direct Load Control through Smart Plugs
}

\author{
Giovanni Neglia ${ }^{1}$, G. Di Bella ${ }^{2}$, L. Giarrè ${ }^{2}$ and I. Tinnirello ${ }^{2}$
}

\begin{abstract}
Balancing energy demand and production is becoming a more and more challenging task for energy utilities also because of the larger penetration of renewable energies which are more difficult to predict and control. While the traditional solution is to dynamically adapt energy production to follow time-varying demand, a new trend is to drive demand itself. Most of the ongoing actions in this direction involve greedy energy consumers, like industrial plants, supermarkets or large buildings. Pervasive communication technologies may allow in the near future to push further the granularity of such approach, by having the energy utility interacting with residential appliances. In this paper we study large scale direct control of inelastic home appliances whose energy demand cannot be shaped, but simply deferred. Our solution does not suppose any particular intelligence at the appliances. The actuators are rather smart plugs-simple devices with local communication capabilities that can be inserted between appliances' plugs and power sockets and are able to interrupt/reactivate power flow through the plug. A simple control message can be broadcast to a large set of smart plugs for probabilistically enabling or deferring the activation requests of a specific load type in order to satisfy a probabilistic bound on the aggregated power consumption. The control law can be easily derived analytically.
\end{abstract}

\section{INTRODUCTION}

Load control in modern power grids is becoming more and more important for maintaining a balance between energy supply and demand. Traditionally, demand was less predictable and less controllable than supply, so that the energy balance was achieved by adapting dynamically generation levels to match the consumption. The increasing penetration of renewable energies has radically changed the scenario, due to their lower predictability. The possibility to control load demand is then becoming more appealing for several actors, such as the energy utilities (which can better plan the production as well as control the grid reliability) and the end customers (who can actively participate to the energy market).

However, despite the many proposals in the literature [1] discussing different demand response programs, load control for residential users (who significantly affect the overall energy load variability [2]) is still limited to pilot projects [3] or is the last resort in critical situations [4]. One of the reasons is that the implementation of user-friendly demand response mechanisms requires often investments (for updating user appliances and communication infrastructure) that are not clearly justified for the end users.

In this paper we consider a solution which enables direct load control for deferrable appliances in a large scale power

\footnotetext{
${ }^{1}$ Giovanni Neglia is with Inria, Maestro project, Sophia Antipolis,France

${ }^{2}$ Giuseppe Di Bella, Laura Giarrè and Ilenia Tinnirello are with Dipartimento di Energia, Ingegneria dell'informazione e Modelli Matematici, Università degli Studi di Palermo, Palermo, Italy.
}

grid, with very limited infrastructure investments for communication and appliances control. Indeed our approach requires no change or limited change to the appliances, because it relies on some devices which can be inserted between the appliances' plugs and the power sockets. These devices are usually called smart plugs and are already produced with a variety of different purposes: prevent vampire power drain, monitor energy usage and generally reduce the overall costs to run various electronics. For our purpose, their basic functionality is to be able to interrupt/reactivate the current flow once they receive a command from a remote controller which may be managed by the energy utility itself or by some other entity like an energy aggregator [5], [6]. In this way the smart plug can simply postpone the appliance's operation reducing instantaneous power demand. The command is relayed to the smart plug from a home gateway, which could be the smart meter or another network element connected to the Internet. The communication between the smart plug and the gateway requires then some local communication protocol, like Ethernet, WiFi, ZigBee, BlueTooth, etc.. The gateway may simply be an application running on a $\mathrm{PC}$, on the ADSL box or even on a smartphone. In many countries a large percentage of the households is already provided with a Local Area Network (often a WiFi one) connected to the Internet. In this case the cost to deploy the solution is basically limited to the cost of the smart plugs. At the moment of writing, there are already commercial devices which can accomplish all the functionalities required and whose price is less than $40 \$$ [7].

Each household is not by itself a greedy energy consumer, then the proposed approach needs a large deployment in order to control a significant percentage of total power demand. Controlling individually each appliance on a fine time scale may require excessive communication signaling overhead as well as computation power at the central controller. Moreover privacy issues may advocate against such form of capillary control. For these reasons we consider an open-loop probabilistic control, which only uses historical information about the aggregate behavior of a group of residential users without the need for bidirectional control messages or high-frequency meter readings. Our mechanism only requires the controller to periodically send a control message, specifying the control policy for a given type of appliances to a group of residential users. The control policy is expressed in terms of the probability that an appliance activation request originated at a given time of the day may be satisfied. Indeed, the activation probability function can be loaded once a day on the gateway.

The counterpart of the absence of a feedback from the 
appliances is that our control cannot provide deterministic guarantees on the total power consumption, but only probabilistic ones. In particular the control signal will be determined in order to assure a maximum probability to exceed a given bound on the power consumption.

This paper is an extension of our previous work appeared in [8]. While the general application scenario is basically the same, the analysis in this paper allows us to determine the optimal control policy under significantly more general assumptions: the power bound can be time-varying, the operation time of each appliance and the delay by which a request is postponed are no more required to be constant, but they may be random variables with general distributions.

The rest of this paper is organized as follows. After a brief literature review in Section II, in Section III we introduce the model and derive the probabilistic control. In Section IV we present some numerical results. Finally, our conclusions are discussed in Section V.

\section{RELATED WORK}

The problem of direct control has been largely studied in literature with several proposals formulating the control mechanism under different optimization objectives, related to the power grid reliability or operation savings. Direct load control is the mechanism allowing electric utilities to turn specific users' appliances on and off during peak demand periods. The usual approach is based on a central controller, working on the basis of dynamic programming optimization [9], fuzzy logic-based decisions [10], or other profit maximization schemes [11]. An admission control mechanism based on the exact knowledge of the total load generated by the controlled users has been proposed in [12].

Recently, real users have been involved in direct load control programs [3], [4]. [3] describes a pilot project where users appliances were modified to react to critical load conditions by reducing their energy demand when the powergrid frequency fell below a given threshold. In the program described in [4] an energy management device, controlled by the energy utility, can switch on and off traditional (unmodified) appliances in critical situations (3-4 times per year). Conversely, the solution we envisage is intended to address a larger set of situations where the utility may want to impose a bound on the aggregate power consumption.

The bandwidth requirements of the control network, as well as the privacy concerns arising in case of continuous monitoring of users' loads, have been addressed in some recent work proposing some simplifications of the optimal control schemes or distributed controllers. For example, in [13], the tradeoff between the importance of exact load characterization (exploration) and control (exploitation) has been analyzed in a restless bandit framework, according to which loads are ranked for their relevance to demandresponse actions. In [14] a distributed controller for a large number of pool pumps is designed on the basis of a Markovian Decision Process model with randomized decisions for avoiding synchronization of pumps. An aggregated model for a large collection of loads operating under the same

\begin{tabular}{|c|c|c|c|c|c|c|c|c|c|c|}
\hline Appliance & 6 & 8 & 10 & 12 & 14 & 16 & 18 & 20 & 22 & 24 \\
\hline Dishwasher. & 3 & 9 & 9 & 3 & 13 & 0 & 16 & 38 & 13 & 3 \\
Laundry m. & 16 & 28 & 38 & 19 & 16 & 19 & 16 & 16 & 3 & 6 \\
\hline
\end{tabular}

TABLE I

APPLIANCE ACTIVATION RATES [\% OVER 30000 USERS]

controller is then broadcast by the utility to all the users for driving the decision process. A similar approach based on a generalized input signal for a group of users and distributed control actions is pursued here but it is applied to deferrable uninterruptible loads.

\section{Model AND CONTROL}

In our scenario the energy utility would like to enforce a time-variant power consumption level $P_{g}(t)$ during a time interval $\left[T_{s}, T_{e}\right]$ for the set of appliances under control. Our solution provides probabilistic guarantees: the instantaneous power consumption $P_{c}(t)$ can exceed $P_{g}(t)$ with probability at most $\epsilon$, i.e., $\operatorname{Prob}\left\{P_{c}(t)>P_{g}(t)\right\} \leq \epsilon$. In what follows we use $*$ to denote the convolution operator: $(f * g)(t)=$ $\int_{-\infty}^{\infty} f(x) g(t-x) \mathrm{d} x$. Moreover, for any random variable $Y$ we denote its probability density function, cumulative distribution function and complementary cumulative distribution function respectively as $f_{Y}, F_{Y}$ and $\bar{F}_{Y}$.

\section{A. Appliance Model}

Our methodology applies to deferrable appliances, whose activation time can be postponed, such as washing machines or laundry machines. We can easily take into account different types of appliances, but in this paper we only consider a single class in order to keep the exposition simple. We assume that the operation time of each appliance is a random variable $D$, and all the operation times are i.i.d.. Similarly the instantaneous power consumption of an appliance is a random variable $X(t)$ with known time-invariant probability density function $f_{X}(x)$. This probabilistic description can easily incorporate the uncertainty about the characteristic of the appliance.

Some statistical studies [15] have characterized the percentage of users activating a specific residential appliance during different intervals of the day. In these studies, the day is divided into equal size intervals and the percentage of active users is averaged in each interval. Table I has been obtained from the data in [15] and shows the percentage of dishwashers/laundry machines active during 2-hour time intervals. Assuming that the user population $U$ is large enough and considering an observation time of one day, we can model the activation instants of a given appliance as a non-homogeneous Poisson process $N(t)$ with arrival rate $\lambda(t)$. For simplicity we consider $N(0)=0$, i.e. there is no appliance active at the reference time $t=0$. For our numerical experiments we used the empirical arrival rate for the laundry machines with 30 minute granularity.

In the absence of any control, the appliances that are active at time $t$ are those turned on at a time $\tau \leq t$ for which 


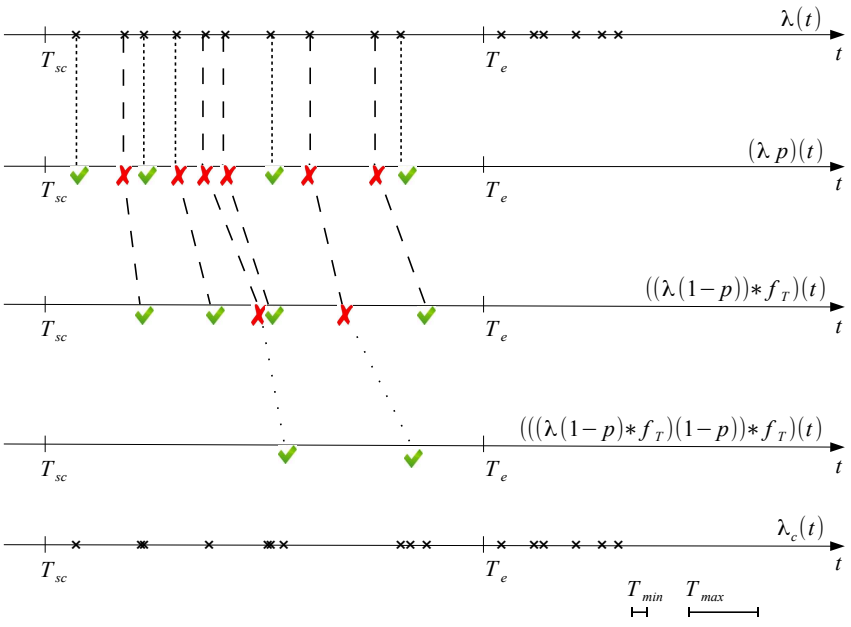

Fig. 1. Example of activation request point processes. The first time axis shows the time instants at which requests are first considered (and when they would be served without the control). The checkmarks in the second, third and fourth time axis respectively correspond to the requests that are immediately satisfied, deferred once and deferred twice. The last time axis shows the aggregate point process of the time instants at which the requests are satisfied.

the operation time $D$ is longer than $t-\tau$. Their number is distributed as a Poisson random variable [16] with parameter $\left(\lambda * \bar{F}_{D}\right)(t)=\int_{0}^{t} \lambda(\tau) \bar{F}_{D}(t-\tau) \mathrm{d} \tau$. Let Pois $(x)$ denote a Poisson random variable with parameter $x$, the instantaneous power consumption $P(t)$ at time $t$ can then be calculated as

$$
P(t)=\sum_{i=1}^{N(t)} X_{i}(t), \text { where } N(t) \sim \text { Pois }\left(\left(\lambda * \bar{F}_{D}\right)(t)\right) .
$$

\section{B. Activation Model under Probabilistic Control}

We propose a control mechanism devised to modify the appliance activation process into a different non-homogeneous Poisson process with rate $\lambda_{c}(t)$, where $\lambda_{c}(t)$ is determined so that the corresponding power consumption $P_{c}(t)$ satisfies the probabilistic constraint imposed by the utility. Observe that $P_{c}(t)$ can be expressed through (1), simply replacing $\lambda(t)$ with $\lambda_{c}(t)$. We first describe our control, i.e., how the arrival rate will be modified from $\lambda(t)$ to $\lambda_{c}(t)$ and then we calculate how to set $\lambda_{c}(t)$.

In our framework the utility applies a time-variant activation probability function $p(t)$. If the user turns on an appliance at time $t$, the appliance will actually start with probability $p(t)$, and with probability $1-p(t)$ the decision about its activation will be postponed by a random variable $T$, whose range is in the interval $\left[T_{\min }, T_{\max }\right]$ with $T_{\min }>$ 0 , i.e. $f_{T}(t)=0$ for $t<T_{\min }$ or $t>T_{\max }$. This simple algorithm is implemented from the smart plug, interposed between the socket and the appliance itself. Observe that the control is not effective when $p(t)=1$. As we are going to show, in order to be able to satisfy the constraint in $\left[T_{s}, T_{e}\right]$, it may be needed to apply the control also before $T_{s}$, i.e. $p(t)<1$ for $t<T_{s}$. We denote $T_{s c}$ the first time instant for which $p(t)<1$ (more formally $T_{s c}=\inf \{t: p(t)<1\}$ ).

The scheme operation is depicted in Fig 1. The first time axis of the figure shows a sample of the point process $(\mathcal{P})$ of the time instants at which the user would like to turn on the appliance. The outcomes of the Bernoulli random variables drawn for every request determine two different point processes that are distinguished in the second time axis by two different marks, corresponding to the requests that are immediately accepted (point process $\mathcal{P}_{a}$ denoted by the checkmarks) and those that are deferred (point process $\mathcal{P}_{d}$ denoted by the crosses). The probability $p(t)$ is determined exogenously and independently from the given sample of the Poisson process $\mathcal{P}$, then both $\mathcal{P}_{a}$ and $\mathcal{P}_{d}$ are (non-homogeneous) Poisson processes respectively with rates $p(t) \lambda(t)$ and $(1-p(t)) \lambda(t)$, and they are independent from each other [17, ch. 4]. The decision about each request in $\mathcal{P}_{d}$ is postponed by $T$ time units as it is shown in the third time axis in Fig. 1. The shifted point process is still a Poisson one [18] with rate

$$
\left((\lambda(1-p)) * f_{T}\right)(t)=\int_{0}^{t} \lambda(\tau)(1-p(\tau)) f_{T}(t-\tau) \mathrm{d} \tau
$$

and independent from $\mathcal{P}_{a}$. At its turn, the shifted point process may be split in two point processes $\mathcal{P}_{d, a}$ and $\mathcal{P}_{d, d}$ respectively of the requests accepted at the second trial or further deferred. A similar reasoning leads to the conclusion that $\mathcal{P}_{d, a}$ and $\mathcal{P}_{d, d}$ are independent Poisson processes with rates respectively $p(t)\left(((1-p) \lambda) * f_{T}\right)(t)$ and $(1-p(t))(((1-$ $\left.p) \lambda) * f_{T}\right)(t)$ and they are also independent from $\mathcal{P}_{a}$.

A request can be deferred at most $K_{\max }=\left\lceil T_{e} / T_{\min }\right\rceil$ times. We can then build $\left\lceil T_{e} / T_{\text {min }}\right\rceil$ independent Poisson processes $\mathcal{P}_{a}, \mathcal{P}_{d, a}, \mathcal{P}_{d, d, a}, \ldots \mathcal{P}_{d, \ldots, d, a}$. Their superposition is still a Poisson process, whose points are the time instants at which the appliances become active. We denote by $\lambda_{c}(t)$ its rate. As we anticipated above, the effect of the probabilistic control is to transform the initial uncontrolled Poisson process $\mathcal{P}$ with rate $\lambda(t)$ into a Poisson process with rate $\lambda_{c}(t)$. All the requests arriving in the interval $\left[0, T_{e}+T_{\max }\right]$ are admitted in the same interval, then it follows that $\int_{0}^{T_{e}+T_{\max }} \lambda(\tau) \mathrm{d} \tau=\int_{0}^{T_{e}+T_{\max }} \lambda_{c}(\tau) \mathrm{d} \tau$.

We can also define the point process $\mathcal{P}_{e q}$ as the sequence of time instants of all the requests, independently from them being accepted or deferred, $\mathcal{P}_{e q}=\mathcal{P}_{a} \cup \mathcal{P}_{d} \cup \mathcal{P}_{d, a} \cup \mathcal{P}_{d, d} \cdots \cup$ $\mathcal{P}_{d, \ldots, d, a} \cup \mathcal{P}_{d, \ldots, d, d}$ whose rate we denote by $\lambda_{e q}(t)$. We observe that $\mathcal{P}_{e q}$ is not in general a Poisson process. The following equation holds for $\lambda_{e q}(t)$ :

$$
\begin{aligned}
\lambda_{e q}(t) & =\lambda(t)+\int_{0}^{t} \lambda_{e q}(\tau)(1-p(\tau)) f_{T}(t-\tau) \mathrm{d} \tau \\
& =\lambda(t)+\left(\left(\lambda_{e q}(1-p)\right) * f_{T}\right)(t) .
\end{aligned}
$$

This equation expresses formally the fact that each activation request is arriving for the first time or it is a previous request rejected at some time $\tau$ (that occurs with probability $1-$ $p(\tau)$ ) and delayed to time $t-\tau$ later. Finally, the rate of the controlled process can be expressed simply as $\lambda_{c}(t)=$ $p(t) \lambda_{e q}(t)$. 


\section{Tuning the Activation Probability Function: the constant bound case}

We first derive our activation probability function for the case when the power bound is constant, i.e. $P_{g}(t)=P_{g}$ for $t \in\left[T_{s}, T_{e}\right]$. We extend the reasoning to the case of a time-variant bound in the following section.

We show how $p(t)$ can be determined to guarantee that $\operatorname{Prob}\left\{P_{c}(t)>P_{g}\right\} \leq \epsilon$ for $t \in\left[T_{s}, T_{e}\right]$. The process $P_{c}(t)$ is completely characterized by the knowledge of the expected number $n_{c}(t)$ of appliances active at time $t\left(n_{c}(t)=\left(\lambda_{c} *\right.\right.$ $\left.\bar{F}_{D}\right)(t)$ ) and the power consumption density of a single appliance $\left(f_{X}().\right)$. By selecting $p(t)$ the utility controls $\lambda_{c}(t)$ and then $n_{c}(t)$.

The first step is to calculate the maximum $n_{c}^{*}$ value that guarantees that $\operatorname{Prob}\left\{P_{c}(t)>P_{g}\right\} \leq \epsilon$. In [8] we develop the calculations for the case when the number of appliances active at a given time instant is large, and the aggregated power consumption can be approximated by a normal distribution. This case is also probably the most relevant from a practical point of view, given that we are interested in controlling a large number of appliances. We obtain that $n_{c}^{*}$ is the largest solution of the following equation:

$$
\left(n_{c}^{*} \mathrm{E}[X]-P_{g}\right)^{2}=z_{1-\epsilon}^{2} n_{c}^{*} \mathrm{E}\left[X^{2}\right],
$$

where $z_{1-\epsilon}$ is the $\epsilon$ percentile of the standard normal distribution.

The second step is then to determine the control $p(t)$ which shapes the controlled rate $\lambda_{c}(t)$ so that $n_{c}(t) \leq n_{c}^{*}$ for each $t \in\left[T_{s}, T_{e}\right]$. While respecting this constraint, we would like $n_{c}(t)$ to be as close as possible to $n_{c}^{*}$, in order to admit as many requests as possible and avoid useless delays. We assume that a smart plug can only block an appliance when the user tries to activate it and not later. Then we need in general to apply the control also before $T_{s}$, otherwise if $n_{c}\left(T_{s}^{-}\right)>n_{c}^{*}$, the constraint would not be satisfied at $T_{s}$. In order to address this situation, we define $n_{c}^{0}(t, \tau)$ to be the expected number of active appliances in the system at time $\tau$ assuming that no other request is accepted after $t$, i.e. when $\lambda_{c}(x)=0$ for $x \in[t, \tau]$. It holds:

$$
n_{c}^{0}(t, \tau)=\int_{0}^{t} \lambda_{c}(x) \bar{F}_{D}(\tau-x) \mathrm{d} x
$$

We observe that $n_{c}^{0}(t, \tau)$ is a non increasing function in $\tau$.

The control $p(t)$ can be determined at each instant $t$, by imposing that $n_{c}^{0}(t, \tau) \leq n_{c}^{*}$ for $\tau \in\left[T_{s}, T_{e}\right]$. Given that we want to minimize the number of requests delayed, $p(t)$ should be as high as possible, while respecting this constraint. It is evident that at a given time $t \in\left[0, T_{e}\right]$ the control should admit new activation requests if $n_{c}^{0}(t, \tau)<n_{c}^{*}$ for $\tau \in\left[t, T_{e}\right]$ and block them if $n_{c}^{0}\left(t, \tau^{\prime}\right)>n_{c}^{*}$ for some $\tau^{\prime} \in\left[t, T_{e}\right]$. The case when $n_{c}^{0}\left(t, \tau^{\prime}\right)=n_{c}^{*}$ for some $\tau^{\prime} \in\left[t, T_{e}\right]$ needs more attention. We need to distinguish when the constraint is met in the future $\left(\tau^{\prime}>t\right)$ or only in the present $\left(\tau^{\prime}=t\right.$ and $n_{c}^{0}(t, \tau)<n_{c}^{*}$ for $\left.\tau>t\right)$. The two cases are shown qualitatively in Fig. 2. If $\tau^{\prime} \neq t$ and $\lambda_{c}(x)=0$ for $x>t$, then it follows from Eq. (3) that

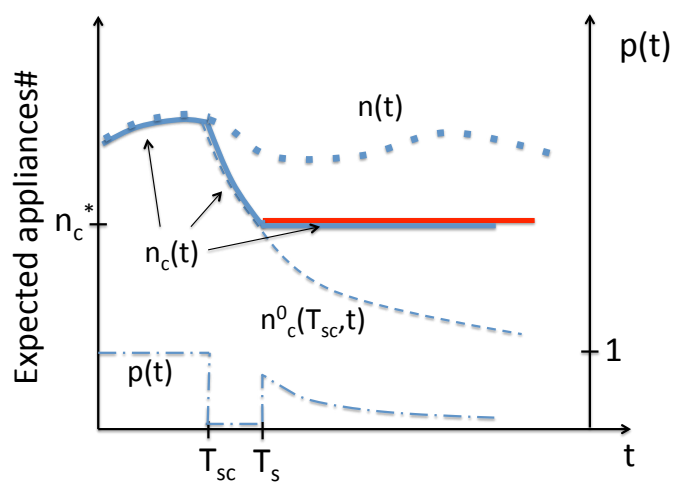

(a)

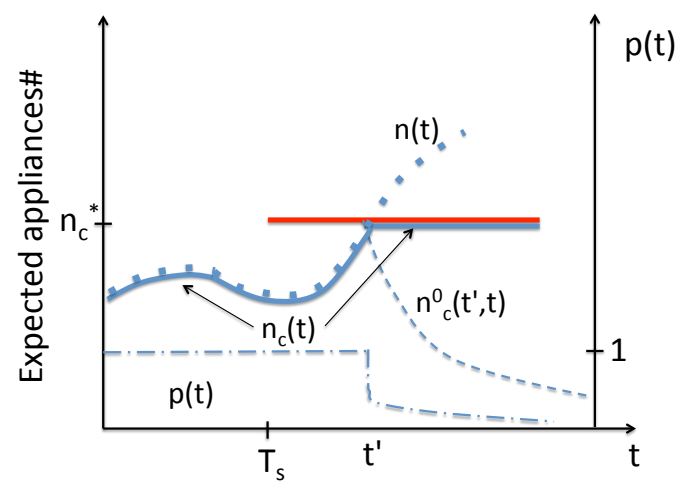

(b)

Fig. 2. Time evolution of the control probability $(p(t)$, dash-dotted curve) and the expected number of active appliance i) in absence of control $(n(t)$, dotted curve), ii) in presence of control $\left(n_{c}(t)\right.$, solid curve), and iii) if no request is accepted after $t\left(n_{c}^{0}(t, \tau)\right.$, dashed curve). Subfigure (a) shows a case where the control is affected by the constraint being hit in the future $\left(p(t)=0\right.$ for $t$ in $\left[T_{s c}, T_{s}\right]$, because $\left.n_{c}^{0}\left(T_{s c}, T_{s}\right)=n_{c}^{*}\right)$. Subfigure (b) shows a case when the control is affected by the constraint being met at present time.

$n_{c}^{0}(\tilde{t}, \tau)=n_{c}^{0}(t, \tau)$ for $\tilde{t} \in\left(t, \tau^{\prime}\right]$ and $\tau>\tilde{t}$. In particular it holds $n_{c}^{0}\left(\tilde{t}, \tau^{\prime}\right)=n_{c}^{0}\left(t, \tau^{\prime}\right)=n_{c}^{*}$ and the constraint is hit even if no new request is admitted. We conclude that if $n_{c}^{0}\left(t, \tau^{\prime}\right)=n_{c}^{*}$ for $\tau^{\prime} \in\left(t, T_{e}\right]$, it is not possible to admit new requests. The constraint may be met in $t$ and only in $t$, i.e. $n_{c}^{0}(t, t)=n_{c}^{*}$ and $n_{c}^{0}(t, \tau)<n_{c}^{*}$ for $\tau>t$. In this case we can potentially admit new requests at the same rate at which working appliances are terminating. This corresponds to equate to zero the derivative of $n_{c}^{0}(t, t)$ :

$$
\lambda_{c}(t) \bar{F}_{D}(0)+\int_{0}^{t} \lambda_{c}(x) \bar{F}_{D}^{\prime}(t-x) \mathrm{d} x=0
$$

from which the target value of $\lambda_{c}($.$) at time t$ can be determined. This target value will actually be reachable if it is smaller than $\lambda_{e q}(t)$. If it is so, then $p(t)$ can be calculated using $\lambda_{c}(t)=p(t) \lambda_{e q}(t)$.

Summarizing the previous discussion, the control law is:

$$
p(t)=\left\{\begin{array}{l}
\min \left(1, \frac{-\int_{0}^{t} \lambda_{c}(x) \bar{F}_{D}^{\prime}(t-x) \mathrm{d} x}{\lambda_{e q}(t) \bar{F}_{D}(0)}\right) \\
\quad \text { if } n_{c}^{0}(t, t)=n_{c}^{*} \wedge n_{c}^{0}(t, \tau)<n_{c}^{*} \forall \tau>t, \\
1 \text { if } n_{c}^{0}(t, \tau)<n_{c}^{*} \quad \forall \tau \geq t, \\
0 \text { otherwise. }
\end{array}\right.
$$


Practically speaking, the utility is going to transmit a discrete time probability function to each appliance (or actuator device).

\section{Tuning the Activation Probability Function: the dynamic bound case}

The analysis above can be easily extended to the case when the upper bound on the total power is time-variant. From $\operatorname{Prob}\left\{P_{c}(t)>P_{g}\right\} \leq \epsilon$ for each $t \in\left[T_{s}, T_{e}\right]$, it is possible to derive the maximum value for the expected number of appliances active at each time instant $t$, that we denote by $n_{c}^{*}(t)$. The optimal control $p(t)$ can then be determined by imposing that, at each time instant $t$, $n_{c}^{0}(t, \tau) \leq n_{c}^{*}(\tau)$ for each $\tau \in\left[T_{s}, T_{e}\right]$, that is at each time $t$ we are sure to be able to satisfy the constraint also in the future. It holds:

$$
p(t)=\left\{\begin{array}{l}
\min \left(1, \frac{-\int_{0}^{t} \lambda_{c}(x) \bar{F}_{D}^{\prime}(t-x) \mathrm{d} x}{\lambda_{e q}(t) \bar{F}_{D}(0)}\right) \\
\quad \text { if } n_{c}^{0}(t, t)=n_{c}^{*}(t) \wedge n_{c}^{0}(t, \tau)<n_{c}^{*}(\tau) \forall \tau>t \\
1 \quad \text { if } n_{c}^{0}(t, \tau)<n_{c}^{*}(\tau) \quad \forall \tau \geq t \\
0 \quad \text { otherwise. }
\end{array}\right.
$$

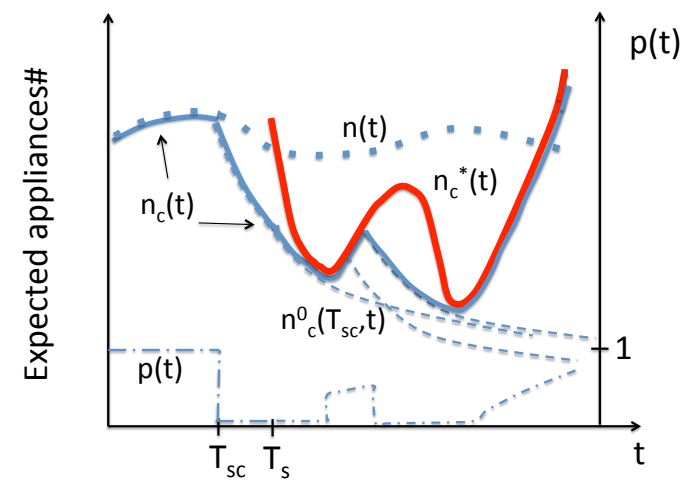

Fig. 3. Time evolution of the control probability $(p(t)$, dash-dotted curve), the dynamic bound on the expected number of active appliances and the expected number of active appliance i) in absence of control $(n(t)$, dotted curve), ii) in presence of control $\left(n_{c}(t)\right.$, solid curve), and iii) if no request is accepted after $t\left(n_{c}^{0}(t, \tau)\right.$, dashed curve).

Fig. 3 shows an example of the qualitative behavior of the optimal control with a dynamic bound. The control will make the expected number of active appliances $n_{c}(t)$ follow as close as possible the maximum value $n_{c}^{*}(t)$, periodically blocking all the requests during some time intervals in order to guarantee that the constraint is not violated in the future. During one of such intervals, say it $\mathcal{I}=(a, b)$, it holds $p(\tau)=0$ and $n_{c}(\tau)=n_{c}^{0}(a, \tau)$ for $\tau \in \mathcal{I}$. The time instant $b$ corresponds to a point of tangency (with coordinates $\left.\left(b, n_{c}^{0}(a, b)\right)\right)$ for the two curves $\left(t, n_{c}^{0}(a, t)\right)$ and $\left(t, n_{c}^{*}(t)\right)$.

\section{A NumericAl EXAMPLE}

As an example we consider the control of a group of 3000 laundry machines, whose activation rates are in Table I. We consider that each laundry machine absorbs a constant power equal to $X=1.5 \mathrm{~kW}$ and has a constant operation time $D=90 \mathrm{~min}$. (this will simplify the interpretation of

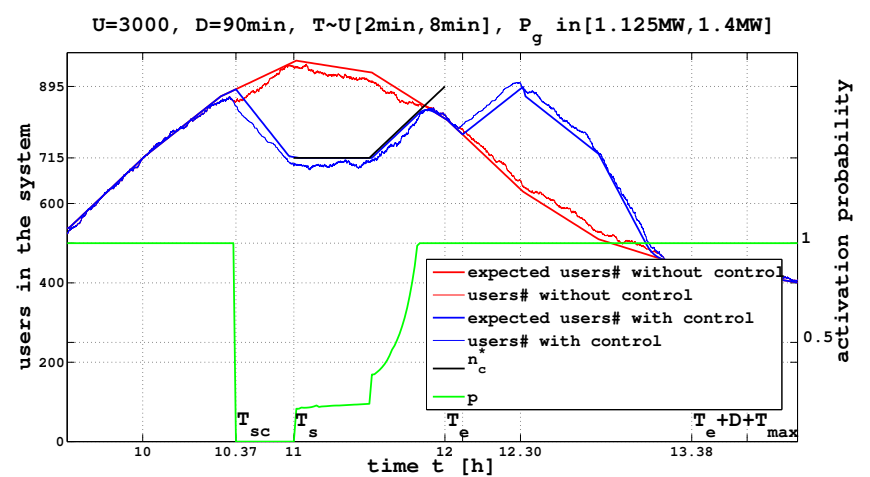

(a)

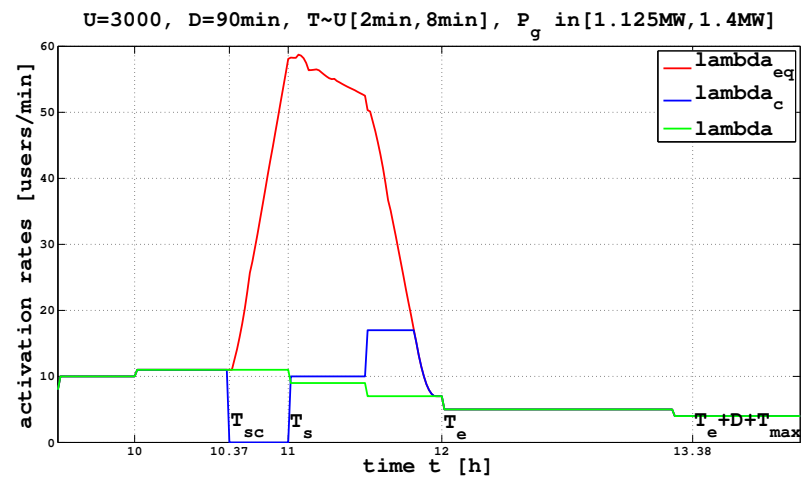

(b)

Fig. 4. Plot (a) shows the expected and actual number of active appliances both under and without control together with the upper bound $n_{c}^{*}(t)$ and the activation probability function $p(t)$. Plot (b) shows the corresponding request rates.

the results). We assume each laundry machine belongs to a different user, hence in what follows we will talk equivalently of users, laundry machines or appliances. In this example $T$ is a random integer uniformly distributed in the range [2min, $8 \mathrm{~min}]$.

Fig. 4 a) shows the expected and actual number of active appliances without control. The maximum expected number of appliances in the considered time interval would be 960 and it would be reached at $11 \mathrm{am}$. This corresponds to an expected aggregate power consumption of $1.440 \mathrm{MW}$.

We consider that the energy utility would like to impose a constraint $P_{g}(t)=1.125 \mathrm{MW}$ between $11 \mathrm{am}$ and 11.30am, this constraint could be violated instantaneously with probability at most $\epsilon=0.1$, from which we obtain (Eq. 2) that $n_{c}^{*}(t)=715$ during this period. Moreover, if no constraint would be imposed after $11.30 \mathrm{am}$, the instantaneous power consumption would show a significant increase immediately after 11.30am due to all the postponed requests that would be immediately accepted. For this reason, the power constraint is linearly increased between 11.30am and 11.59am up to the value 1.4 MW. Correspondingly $n_{c}^{*}(t)$ increases from 715 to 895.

The optimal control $p(t)$ can be calculated from Eq. 5 and it is shown in Fig. 4 a) together with the corresponding expected and actual number of active appliances. We observe that all the requests are admitted $(p(t)=1)$ up to time $T_{s c}=$ 
10.37am and then the two processes with and without control overlap. Starting from $T_{s c}$, no request is accepted until $T_{s}=$ $11 \mathrm{am}$ and indeed the expected number of active appliances decreases and hits the constraint at $T_{s}$. Fig. 4 b) shows how, during this interval, the rate at which requests are admitted $\left(\lambda_{c}\right)$ is indeed 0 and the rate at which requests arrive $\left(\lambda_{e q}\right)$ increases significantly. Starting from $T_{s}$, new appliances are admitted with some probability at the same rate at which previously admitted ones terminate until 11.30am. During this interval the corresponding rate $\lambda_{c}$ is slightly higher (10 requests per minute) than the rate of requests arriving for the first time $\lambda$ ( 9 requests per minute). The system is then able to satisfy some of the requests in the backlog, and the total request rate $\lambda_{e q}$ decreases. From $11.30 \mathrm{am}$ to $11.59 \mathrm{am}$, the expected number of active appliances can increase. This corresponds first to a jump in the rate $\lambda_{c}$ at which new appliances can be admitted (18 requests per minute) and a remarkable decrease in the total request rate $\lambda_{e q}$. While a rate of 18 requests per minute is compatible with the upper bound, as soon as $\lambda_{e q}$ becomes smaller than such value, the rate of requests admitted has necessarily to decrease too. And this is shown to happen in Fig. $4 \mathrm{~b}$ ) around 11.55am. From this instant up to $11.59 \mathrm{am}$ it holds $\lambda_{e q}=\lambda_{c}$ and $p(t)=1$. At 11.58am finally the backlog is exhausted and $\lambda_{e q}=\lambda_{c}=\lambda$. The control periods ends at 11.59am and these rates will not differ anymore, that means that the total number of requests admitted up to time $t$ will be the same for $t \geq 11.58 \mathrm{am}$.

While nothing seems to happen after 11.58am from Fig. 4 b), Fig. 4 a) shows an increase in the number of active appliances a few minutes after the end of the control period (starting from $12.07 \mathrm{pm}$ exactly) that can appear unexpected at a first look, specially if compared with the corresponding curve for the uncontrolled case. We explain this observation as follows. By time 11.58am the same number of appliances has been activated both in presence and in absence of the control. What is different is that they have been activated later in presence of the control. In particular many appliances start working between 10.37am and 11.00am in the uncontrolled case, while no appliance starts during the same period in the controlled case. In the first case these appliances become inactive $90 \mathrm{~min}$. later, i.e. between $12.07 \mathrm{am}$ and $12.30 \mathrm{am}$. On the contrary in presence of the control, there is no appliance terminating during such period, while new requests are accepted. For this reason we observe a steady increase in the number of active appliances until 12.30. With similar considerations we can explain how the number of active appliances evolves for later times.

This example shows how load shifting of appliances with long operation time can have consequences well after the end of the interval the control is applied. It is then important to correctly shape the power bound for long time intervals.

\section{CONClusions And Future Works}

The role of direct load control in modern power grids has been shown to be beneficial for several applications. However, in the case of small individual energy loads, these benefits can be appreciable only if a large number of users are involved in the control process. The main contribution of this paper is proposing a load control mechanism whose deployment requires minimal communication overhead in order to allow a large scale deployment. The idea is to work on deferrable loads whose activation requests are admitted by a local energy controller on the basis of a probabilistic admission function. This function is periodically signaled by the energy utility according to the expected load demand and desired power limit. In the current scheme, we assume that the expected load demand is simply characterized by collecting historical data, quantifying the appliance arrival rate in different intervals of the day, and assuming that these rates do not change day by day. An interesting model extension, that we are considering as a future work, is coupling the proposed control scheme with a mechanism for estimating the actual time-varying arrival rate of activation requests from the instantaneous aggregated load in a privacypreserving way.

\section{REFERENCES}

[1] V. S. K. M. Balijepalli, V. Pradhan, S. A. Khaparde, and R. M. Shereef, "Review of Demand Response under Smart Grid Paradigm," in IEEE PES Innovative Smart Grid Technologies, 2011.

[2] "Buildings energy data book." [Online]. Available: http://buildingsdatabook.eren.doe.gov/default.asp

[3] D. J. Hammerstrom, " Pacific Northwest GridWise Testbed Demonstration Projects Part II. Grid Friendly Appliance Project," Pacific Northwest, Tech. Rep. PNNL 17079, 2007.

[4] "FPL on call saving program." [Online]. Available: www.fpl.com/residential/energy_saving/programs/oncall.shtml

[5] A. Abdisalaam, I. Lampropoulos, J. Frunt, G. Verbong, and W. Kling, "Assessing the economic benefits of flexible residential load participation in the dutch day-ahead auction and balancing market," in European Energy Market (EEM), 2012 9th International Conference on the, 2012, pp. 1-8.

[6] O. Baud, "Energy-pool," March 2013, http://www.energypool.eu/index.php?lang=en.

[7] "Smartplug," https://www.alertme.com/products/smartplug-1622.html.

[8] G. Neglia, G. Di Bella, L. Giarré, and I. Tinnirello, "Unidirectional Probabilistic Direct Control for Deferrable Loads," in Proc. of the 33rd IEEE International Conference on Computer Communications (INFOCOM), workshop on Communications and Control for Smart Energy Systems, 2014.

[9] Y. Y. Hsu and C. C. Su, "Dispatch of direct load control using dynamic programming," IEEE Transactions on Power Systems, vol. 6, no. 3, p. $10561061,1991$.

[10] K. Bhattacharyya and M. L. Crow, "A fuzzy logic based approach to direct load control," IEEE Transactions on Power Systems, vol. 11, no. 2, pp. 708-714, 1996.

[11] G. B. Sheble and K. H. Ng, "Direct load control-a profit-based load management using linear programming," IEEE Transactions on Power Systems, vol. 13, no. 2, pp. 668-694, 1998.

[12] G. Di Bella, L. Giarré, M. Ippolito, A. Jean-Marie, G. Neglia, and I. Tinnirello, "Modeling Energy Demand Aggregator for Residential Users," in IEEE CDC, 2013.

[13] J. A. Taylor and J. L. Mathieu, "Index Policies for Demand Response Under Uncertainty," in IEEE CDC, 2013.

[14] S. P. Meyn, P. Barooah, A. Busić, and J. Ehren, "Ancillary Service to the Grid from Deferrable Loads: The Case for Intelligent Pool Pumps in Florida," in IEEE CDC, 2013.

[15] R. Miceli, "Sustainable Development and Energy Saving Laboratory," DIEET - University of Palermo, Tech. Rep., August 2007.

[16] S. G. Eick, W. A. Massey, and W. Whitt, "The physics of the Mt/G/ $\infty$ queue," Operations Research, vol. 41, no. 4, pp. 731-742, 1993.

[17] D. R. Cox and V. Isham, Point processes. CRC Press, 1980, vol. 12.

[18] S. G. Eick, W. A. Massey, and W. Whitt, "The physics of the $M_{t} / G / \infty$ queue," Operations Research, vol. 41, no. 4, pp. 731-742, July-August 1993. 